Pesq. Vet. Bras. 30(11):968-973, novembro 2010

\title{
Prevalência de neoplasmas cutâneos em cães da Região Metropolitana de Porto Alegre, RS: 1.017 casos $(2002-2007)^{1}$
}

\author{
Adriana E.W.B. Meirelles², Eduardo C. Oliveira², Berenice A. Rodrigues ${ }^{3}$, \\ Giovana R. Costa ${ }^{2}$, Luciana Sonne ${ }^{2}$, Elisa S. Tesser ${ }^{2}$ e David Driemeier ${ }^{2 *}$
}

\begin{abstract}
Meirelles A.E.W.B., Oliveira E.C., Rodrigues B.A., Costa G.R., Sonne L., Tesser E.S. \& Driemeier D. 2010. [Prevalence of cutaneous neoplasms in dogs from the metropolitan area of Porto Alegre, RS, Brazil: 1,017 cases (2002-2007).] Prevalência de neoplasmas cutâneos em cães da Região Metropolitana de Porto Alegre, RS: 1.017 casos (2002-2007). Pesquisa Veterinária Brasileira 30(11):968-973. Setor de Patologia Veterinária, Faculdade de Veterinária, Universidade Federal do Rio Grande do Sul, Av. Bento Gonçalves 9090, Porto Alegre, RS 91540-000, Brazil. E-mail: davetpat@ ufrgs.br.

The aim of this study was to perform a retrospective study of cutaneous neoplasms diagnosed in dogs. The evaluation was established by analyzing the diagnostic files at the Veterinary Pathology Sector, UFRGS, Brazil, over a 6-year period (2002 to 2007). During this period a total of 1869 (37.3\%) skin samples were obtained from 5016 different tissue samples of dogs submitted for examination. Among the referred skin samples, 1002 were from dogs with the diagnosis of cutaneous neoplasia and 15 dogs exhibited more than one type of skin tumor, what amounted to a total of $1017(20.3 \%)$ cutaneous tumor samples. Results confirmed $50.5 \%$ (514/1017), $45.1 \%$ (459/1017), and 3.9\% (40/1017) of respectively mesenquimal, epithelial, and melanocytic origin. Mast cell tumor was the most frequent neoplasia, diagnosed in 228 cases $(22.4 \%)$, and was followed by squamous cell carcinoma (7.5\%), lipoma (7.3\%), perianal gland adenoma (7.1\%), and trichoblastoma (5.8\%). Purebred dogs such as Cocker Spaniel, Boxer, Poodle and German Sheepdog were the most representative breeds affected by various neoplasms. The data obtained, compared to data from previous studies, emphasize the variables breed, age and sex related to some skin tumors, and reinforce the importance and prevalence of different types of skin tumors in dogs.
\end{abstract}

INDEX TERMS: Cutaneous neoplasms, dogs.

RESUMO.- O objetivo deste trabalho foi realizar um estudo retrospectivo sobre neoplasias cutâneas diagnosticadas em cães. A avaliação foi realizada pela análise dos arquivos diagnósticos do Setor de Patologia Veterinária (SPV) da Universidade Federal do Rio Grande do Sul (UFRGS), Brasil, considerando-se um intervalo de seis anos (2002 a 2007). Neste intervalo, um total de 1.869 (37,3\%) amostras de pele canina foram obtidas de 5.016 amostras

\footnotetext{
${ }^{1}$ Recebido em 3 de maio de 2010.

Aceito para publicação em 20 de agosto de 2010

2 Setor de Patologia Veterinária, Faculdade de Veterinária, Universidade Federal do Rio Grande do Sul (UFRGS), Av. Bento Gonçalves 9090, Porto Alegre, RS 91540-000, Brasil. *Autor para correspondência: davetpat@ufrgs.br

${ }^{3}$ Médica Veterinária autônoma, Av. Cristo Rei 134, São Leopoldo, RS 93020-350, Brasil.
}

variadas de tecidos de cães encaminhadas ao SPV. Dentre as amostras cutâneas, 1.002 pertenciam a cães diagnosticados com um tipo de neoplasia cutânea e 15 animais apresentaram mais de uma neoplasia de pele, totalizando $1.017(20,3 \%)$ amostras. Os resultados revelaram que 50,5\% (514/1017) das neoplasias cutâneas apresentaram origem mesenquimal, $45,1 \%(459 / 1017)$ para epitelial e 3,9\% (40/1017) para melanocítica. Mastocitoma foi o tipo neoplásico cutâneo mais frequente, diagnosticado em 228 casos $(22,4 \%)$, seguido por carcinoma de células escamosas $(7,5 \%)$, lipoma $(7,3 \%)$, adenoma de glândula perianal $(7,1 \%)$ e tricoblastoma $(5,8 \%)$. Cocker Spaniel, Boxer, Poodle e Pastor Alemão foram as raças mais representadas em diversos neoplasmas. Os dados obtidos, comparados aos estudos prévios, ressaltam as variáveis raças, idade e sexo, relacionadas a alguns tumores cutâ- 
neos e salientam a importância e prevalência dos diferentes tipos de neoplasia cutânea em cães.

TERMOS DE INDEXAÇÃO: Neoplasias cutâneas, cães.

\section{INTRODUÇÃO}

Os tumores cutâneos são os neoplasmas mais frequentemente encontrados nos animais domésticos. A prevalência nesta região do corpo deve-se ao fato de que a pele, além de ser o maior de todos os órgãos, é constituída, juntamente com o tecido subcutâneo, por uma grande variedade de células suscetíveis ao desenvolvimento de neoplasias (Stannard \& Pulley 1978). A pele tem um alto índice de renovação celular, o que aumenta as chances de ocorrência de mutações, e pode propiciar o surgimento de tumores (Murphy 2006a). Além disso, é um órgão que com frequência é diretamente exposto a condições potencialmente oncogênicas; um exemplo é a exposição aos raios solares, que pode agir como fator predisponente para tumores como o carcinoma de células escamosas (Medleau \& Hnilica 2003) e o hemangiossarcoma (Gross et al. 2005).

O conhecimento da anatomia da pele é importante para permitir a diferenciação entre tumores primários e metástases cutâneas (Murphy 2006a). Os neoplasmas que ocorrem na pele podem ter origem ectodérmica (epiderme e seus anexos), mesodérmica (elementos estruturais da derme, tais como tecido fibroso, tecido adiposo, vasos sanguíneos e músculos, e aqueles relacionados a células hematopoéticas da derme, tais como histiócitos, mastócitos, linfócitos e plasmócitos) ou podem pertencer à categoria dos tumores melanocíticos, formado por melanócitos com origem em células neuroectodérmicas a partir de melanoblastos que migraram da crista neural para a camada basal da epiderme (Yager \& Scott 1992, Gross et al. 2005, Murphy 2006a).

Mastocitoma, lipoma, adenoma perianal, carcinoma de células escamosas, tricoblastoma e histiocitoma são alguns dos tumores cutâneos mais frequentes em cães (Rothwell 1987, Dobson et al. 2002, Kaldrymidou et al. 2002, Bellei et al. 2006, Murphy 2006b, Souza et al. 2006, Pakhrin et al. 2007).

O presente trabalho teve por objetivo avaliar a prevalência e os dados gerais de cães acometidos por neoplasmas cutâneos de amostras enviadas ao Setor de Patologia Veterinária (SPV) da Universidade Federal do Rio Grande do Sul (UFRGS), Porto Alegre, e relacioná-los com outros estudos.

\section{MATERIAL E MÉTODOS}

Para o estudo foram analisados os arquivos de resultados de biópsias enviadas ao Setor de Patologia Veterinária (SPV) da Universidade Federal do Rio Grande do Sul (UFRGS) referentes ao período de 2002-2007. As amostras eram procedentes de clínicas e hospitais veterinários da Região Metropolitana de Porto Alegre, Rio Grande do Sul, Brasil. Os dados analisados incluíram diagnóstico de neoplasmas cutâneos (primários e secundários), prevalência de raça, sexo e idade dos cães. Não foram considerados neste levantamento neoplasmas mamários, orais, genitais e nódulos cutâneo não-neoplásicos, como, por exemplo, as hiperplasias sebáceas nodulares e cis- tos. Ao incluir casos de tumor venéreo transmissível (TVT), somente os cutâneos foram considerados. Os tipos neoplásicos apresentados foram separados em epiteliais, mesenquimais e melanocíticos conforme Goldschmidt et al. (1998) que se baseou na International Histological Classification of Tumors of Domestic Animals World Health Organization (WHO). A prevalência de tumores benignos e malignos foi determinada. Foram contabilizadas as porcentagens de amostras de pele e de neoplasmas cutâneos nas diferentes raças. Nas raças em que a prevalência do neoplasma cutâneo foi alta, observaram-se separadamente os tipos tumorais mais frequentes.

\section{RESULTADOS}

No período de 2002-2007, o Setor de Patologia Veterinária (SPV), UFRGS, recebeu amostras teciduais diversas de 5.016 cães. Destas, 1.869 (37,3\%) eram amostras de pele. Um total de 1.002 cães foi diagnosticado com neoplasia cutânea. Como 15 destes cães apresentavam dois tipos diferentes de tumor de pele, o número total de amostras de tumor de pele somou $1.017(20,3 \%)$.

Nos registros analisados, 53,1\% (533/1.004) dos cães com neoplasma cutâneo eram machos, 45,2\% (454/1.004) fêmeas e em $1,7 \%$ o sexo não foi informado no histórico. Na população total de cães analisada, 35,1\% (1.756/5.016) eram machos, $61,5 \%$ (3.084/5.016) fêmeas e em 3,5\% o sexo não foi mencionado nos registros.

Quanto ao sítio de origem da neoplasia, 50,5\% (514/1.017) dos casos eram de origem mesenquimal, $45,1 \%$ (459/1.017) epitelial, 3,9\% (40/1.017) melanocítica e 0,4\% (4/1.017) apresentaram característica simultânea de mesenquimal e epitelial. Neoplasmas cutâneos malignos foram diagnosticados em 62,3\% (634/1.017) cães e benignos em 37,7\% (383/1.017).

Quarenta e quatro tipos diferentes de neoplasmas cutâneos foram diagnosticados nas amostras enviadas durante o período considerado neste levantamento (Quadro 1). Mastocitoma, carcinoma de células escamosas, lipoma, adenoma de glândula perianal e tricoblastoma foram os tumores mais diagnosticados, representando 50,0\% (508/ 1.017) do total de neoplasmas cutâneos em cães.

Neste estudo, o neoplasma diagnosticado com mais frequência foi o mastocitoma (228/1.017 [22,4\%]). Desses, 103 eram machos, 122 eram fêmeas e três cães não tiveram o sexo informado nos protocolos. A idade média dos cães afetados foi de 7,6 anos, com variação de 8 meses a 19 anos. Cães sem raça definida (SRD) tiveram a maior incidência deste tumor seguidos dos cães das raças Boxer e Cocker Spaniel. Observou-se também a classificação de Patnaik et al. (1984), mastocitoma de grau I abrangeu 59 amostras $(25,9 \%)$, de grau Il abrangeu 118 amostras $(51,8 \%)$ e de grau III abrangeu 51 amostras $(22,4 \%)$.

O carcinoma de células escamosas (ou carcinoma epidermoide) representou o segundo neoplasma mais frequente, encontrado em $76(7,45 \%)$ cães. A idade média dos cães acometidos foi de 8,8 anos, com variação de 2 a 20 anos. Dentre os cães de raça, Pastor Alemão e Rottweiler foram as mais prevalentes.

O lipoma foi o terceiro tumor mais descrito, encontrado em $74(7,3 \%)$ cães. A média da idade dos cães com lipoma 
Quadro 1. Distribuição dos neoplasmas cutâneos caninos diagnosticados pelo SPV da UFRGS (2002-2007)

\begin{tabular}{l}
\hline Classificação histopatológica \\
\hline Mastocitoma \\
Carcinoma de células escamosas \\
Lipoma \\
Adenoma perianal \\
Tricoblastoma \\
Carcinoma apócrino \\
Carcinoma perianal \\
Histiocitoma cutâneo canino \\
Adenoma sebáceo \\
Melanoma \\
Tumor mesenquimal maligno \\
Hemangioma \\
Fibrossarcoma \\
Hemangiossarcoma \\
Hemangiopericitoma canino \\
Epitelioma perianal \\
Acantoma ceratinizante infundibular \\
Adenoma meibomiano \\
Plasmocitoma \\
Tumor venéreo transmissível \\
Epitelioma sebáceo \\
Carcinoma de células basais \\
Sarcoma histiocítico \\
Fibroma \\
Papiloma \\
Adenoma apócrino \\
Lipossarcoma \\
Melanocitoma \\
Carcinoma sebáceo \\
Fibropapiloma \\
Tumor maligno de bainha de nervo periférico \\
Tricoepitelioma \\
Carcinoma ceruminoso \\
Carcinoma anaplásico mamário (metástase na pele) \\
Epitelioma meibomiano \\
Pilomatricoma \\
Carcinoma meibomiano \\
Carcinossarcoma mamário (metástase na pele) \\
Fibrolipoma \\
Carcinoma basoescamoso \\
Linfangiossarcoma \\
Linfoma \\
Mixoma \\
\hline Total glômico maligno \\
Tumor
\end{tabular}

Número \%

$228 \quad 22,4$

$76 \quad 7,5$

$74 \quad 7,3$

$72 \quad 7,1$

$59 \quad 5,8$

$45 \quad 4,4$

$41 \quad 4,0$

$39 \quad 3,8$

$38 \quad 3,7$

$35 \quad 3,4$

$32 \quad 3,2$

$31 \quad 3,0$

303,0

$27 \quad 2,6$

$17 \quad 1,7$

$15 \quad 1,5$

$14 \quad 1,4$

$14 \quad 1,4$

$14 \quad 1,4$

$12 \quad 1,2$

$11 \quad 1,1$

$10 \quad 1,0$

$10 \quad 1,0$

$9 \quad 1,0$

$8 \quad 0,8$

$6 \quad 0,6$

$6 \quad 0,6$

$5 \quad 0,5$

$4 \quad 0,4$

$4 \quad 0,4$

$4 \quad 0,4$

$4 \quad 0,4$

30,3

$3 \quad 0,3$

$3 \quad 0,3$

$3 \quad 0,3$

$2 \quad 0,2$

20,2

$2 \quad 0,2$

10,1

$1 \quad 0,1$

10,1

10,1

$1 \quad 0,1$

$1.017 \quad 100$

foi de 8,7 anos, com variação de 2 a 15 anos. As raças mais afetadas por esse neoplasma foram Cocker Spaniel e Poodle.

$\mathrm{O}$ quarto tumor mais diagnosticado foi o adenoma perianal, observado em $72(7,1 \%)$ animais. A média de idade neste grupo foi de 10,3 anos, com variação entre 2,5 e 20 anos. Um dado que se destaca é a porcentagem de machos $(85,9 \%)$ diagnosticados com este tumor. A frequência elevada em machos também foi encontrada nos casos de carcinoma de glândula perianal $(90,5 \%)$ e epitelioma perianal (100\%). Encontrou-se uma maior prevalência de adenoma perianal em cães SRD, seguida pela observada em cães das raças Poodle e Cocker Spaniel.

Tricoblastoma (incluindo os previamente denominados tumores de células basais) foram vistos em $59(5,8 \%)$ cães. A média de idade foi de 8,3 anos, com variação de 2,4 a
Quadro 2. Média da idade (anos), sexo e raça de cães acometidos pelos principais tumores cutâneos diagnosticados pelo SPV da UFRGS (2002-2007)

\begin{tabular}{|c|c|c|c|}
\hline $\begin{array}{l}\text { Classificação } \\
\text { histopatológica }\end{array}$ & $\begin{array}{l}\text { Média da } \\
\text { idade } \\
\text { (anos) }\end{array}$ & $\begin{array}{l}\text { Sexo } \\
(\%)\end{array}$ & $\begin{array}{c}\text { Raça } \\
(\%)\end{array}$ \\
\hline Mastocitoma & 7,6 & $\begin{array}{l}M: 45,2 \\
F: 53,5 \\
N I: 1,3\end{array}$ & $\begin{array}{c}\text { SRD }(30,4) \\
\text { Boxer }(27,7) \\
\text { Cocker Spaniel }(5,9)\end{array}$ \\
\hline $\begin{array}{l}\text { Carcinoma de } \\
\text { células escamosas }\end{array}$ & 8,8 & $\begin{array}{l}\mathrm{M}: 50,0 \\
\mathrm{~F}: 47,4 \\
\mathrm{NI}: 2,6\end{array}$ & $\begin{array}{c}\text { SRD }(21,0) \\
\text { Pastor Alemão }(10,5) \\
\text { Rottweiler }(9,2)\end{array}$ \\
\hline Lipoma & 8,7 & $\begin{array}{l}\mathrm{M}: 27,0 \\
\mathrm{~F}: 68,9 \\
\mathrm{NI}: 4,1\end{array}$ & $\begin{array}{c}\operatorname{SRD}(27,1) \\
\text { Poodle }(10,0) \\
\text { Cocker }(10,0)\end{array}$ \\
\hline Adenoma perianal & 10,3 & $\begin{array}{l}M: 86,1 \\
F: 13,9\end{array}$ & $\begin{array}{c}\operatorname{SRD}(33,8) \\
\text { Poodle }(15,5) \\
\text { Cocker Spaniel }(12,7)\end{array}$ \\
\hline Tricoblastoma & 8,4 & $\begin{array}{l}M: 58,2 \\
F: 41,8\end{array}$ & $\begin{array}{c}\text { Poodle }(32,8) \\
\text { SRD }(14,9) \\
\text { Cocker Spaniel }(11,9)\end{array}$ \\
\hline Carcinoma perianal & 10,6 & $\begin{array}{l}\text { M: } 92,7 \\
F: 7,3 \\
N I: 2,4\end{array}$ & $\begin{array}{c}\operatorname{SRD}(39,0) \\
\text { Poodle }(22,0) \\
\text { Cocker Spaniel }(9,8)\end{array}$ \\
\hline $\begin{array}{l}\text { Histiocitoma cutâneo } \\
\text { canino }\end{array}$ & 2,6 & $\begin{array}{l}M: 53,8 \\
F: 46,2\end{array}$ & $\begin{array}{c}\text { Cocker Spaniel }(15,4) \\
\text { Boxer }(12,8) \\
\text { Beagle }(10,3)\end{array}$ \\
\hline Adenoma sebáceo & 9,5 & $\begin{array}{l}M: 60,5 \\
\mathrm{~F}: 36,8 \\
\mathrm{NI}: 2,6\end{array}$ & $\begin{array}{c}\text { Poodle }(26,3) \\
\text { SRD }(15,8) \\
\text { Cocker Spaniel }(7,9)\end{array}$ \\
\hline Melanoma & 9,3 & $\begin{array}{l}M: 62,9 \\
F: 31,4 \\
N I: 5,7\end{array}$ & $\begin{array}{c}\text { Cocker Spaniel(20,0) } \\
\operatorname{SRD}(17,1) \\
\text { Dachshund }(11,4)\end{array}$ \\
\hline Hemangioma & 7,8 & $\begin{array}{l}M: 51,6 \\
F: 48,4\end{array}$ & $\begin{array}{c}\operatorname{SRD}(19,4) \\
\text { Poodle }(12,9) \\
\text { Pastor Alemão(9,7) }\end{array}$ \\
\hline Fibrossarcoma & 8,7 & $\begin{array}{l}\mathrm{M}: 50,0 \\
\mathrm{~F}: 0,0\end{array}$ & $\begin{array}{c}\operatorname{SRD}(36,7) \\
\text { Rottweiler(20,0) } \\
\text { Cocker Spaniel }(10,0)\end{array}$ \\
\hline Hemangiossarcoma & 8,7 & $\begin{array}{l}M: 74,1 \\
F: 25,9\end{array}$ & $\begin{array}{c}\operatorname{SRD}(37,0) \\
\text { Boxer }(18,5) \\
\text { Dogue Alemão }(14,8)\end{array}$ \\
\hline $\begin{array}{l}\text { Hemangiopericitoma } \\
\text { canino }\end{array}$ & 9,1 & $\begin{array}{l}M: 64,7 \\
F: 35,3\end{array}$ & $\begin{array}{c}\operatorname{SRD}(47,1) \\
\text { Boxer }(11,8)\end{array}$ \\
\hline Epitelioma perianal & 12,4 & M:100 & $\begin{array}{c}\text { SRD }(33,3) \\
\text { Cocker Spaniel }(26,7)\end{array}$ \\
\hline $\begin{array}{l}\text { Acantoma ceratinizante } \\
\text { infundibular }\end{array}$ & 6,1 & $\begin{array}{l}M: 35,7 \\
F: 50,0 \\
\mathrm{NI}: 14,3\end{array}$ & $\begin{array}{l}\text { Poodle }(28,57) \\
\text { Pastor Alemão }(21,43) \\
\text { Yorkshire }(14,29)\end{array}$ \\
\hline Plasmocitoma & 8,3 & $\begin{array}{l}M: 50,0 \\
F: 50,0\end{array}$ & $\begin{array}{c}\operatorname{SRD}(35,7) \\
\text { Boxer/Cocker }(21,4)\end{array}$ \\
\hline Adenoma meibomiano & 9,8 & $\begin{array}{l}M: 46,2 \\
F: 53,8\end{array}$ & SRD/Cocker $(23,1)$ \\
\hline $\begin{array}{l}\text { Tumor venéreo } \\
\text { transmissível }\end{array}$ & 4,6 & $\begin{array}{l}M: 66,7 \\
F: 33,3\end{array}$ & $\begin{array}{c}\text { SRD }(58,3) \\
\text { Pinscher }(16,7) \\
\text { Pastor Alemão/ } \\
\text { Poodle }(8,3)\end{array}$ \\
\hline
\end{tabular}

$\mathrm{F}$ = fêmea; M: macho; $\mathrm{NI}$ = não informado; $\mathrm{SRD}$ = sem raça definida.

15 anos. A raça Poodle foi a mais frequentemente acometida, seguida pelos Cocker Spaniels.

Dados sobre os principais neoplasmas encontrados, incluindo idade média, sexo e raças afetadas por tumores cutâneos estão no Quadro 2. Nesse quadro também podese observar a prevalência nos cães sem raça definida (SRD). 


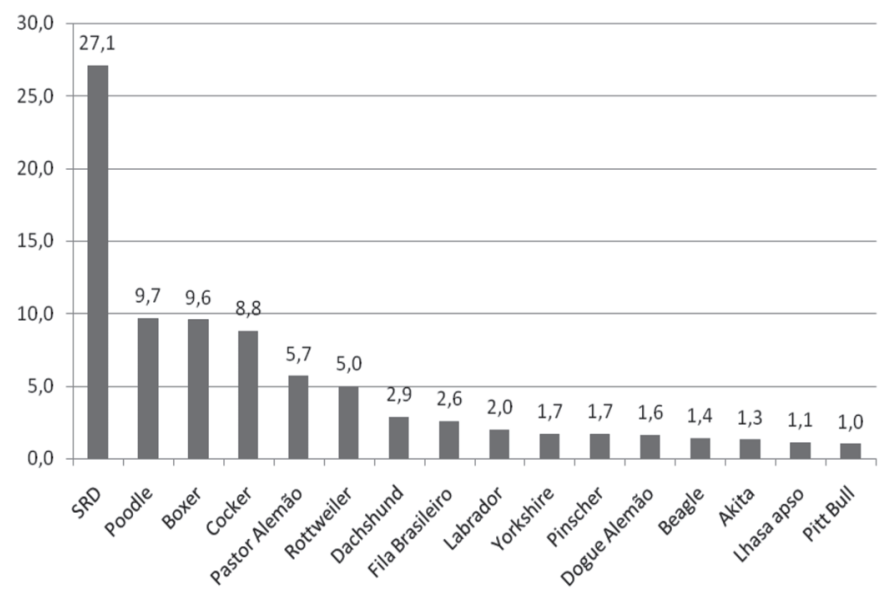

Fig.1. Distribuição porcentual das raças caninas com neoplasmas cutâneos diagnosticados pelo SPV da UFRGS, Porto Alegre, RS (2002-2007).

\section{Quadro 3. Número de cães, idade média (anos), sexo e porcentagem dos neoplasmas cutâneos mais encontrados nas três raças mais registradas entre 2002 e 2007 no SPV da UFRGS}

\begin{tabular}{|c|c|c|c|c|c|}
\hline Raça & $\begin{array}{c}\text { № } \\
\text { de } \\
\text { animais }\end{array}$ & $\begin{array}{l}\text { Idade } \\
\text { média } \\
\text { (anos) }\end{array}$ & $\begin{array}{l}\text { Sexo } \\
(\%)\end{array}$ & $\begin{array}{l}\text { Classificação } \\
\text { histopatológica }\end{array}$ & $\begin{array}{c}\text { Porcentagem } \\
(\%)\end{array}$ \\
\hline \multirow{7}{*}{ Poodle } & 97 & 9,9 & M:61,86 & Tricoblastoma & 22,7 \\
\hline & & & $F: 37,11$ & Adenoma perianal & 11,3 \\
\hline & & & $\mathrm{NI}: 1,03$ & Adenoma sebáceo & 10,3 \\
\hline & & & & Carcinoma perianal & 9,3 \\
\hline & & & & Lipoma & 7,2 \\
\hline & & & & Mastocitoma & 6,2 \\
\hline & & & & Hemangioma & 4,1 \\
\hline \multirow[t]{6}{*}{ Boxer } & 96 & 6,5 & M:44,79 & Mastocitoma & 63,5 \\
\hline & & & $F: 54,17$ & $\begin{array}{c}\text { Histiocitoma cutâneo } \\
\text { canino }\end{array}$ & 5,2 \\
\hline & & & NI:1,04 & Hemangiossarcoma & 5,2 \\
\hline & & & & CCE & 4,2 \\
\hline & & & & Plasmocitoma & 3,1 \\
\hline & & & & Melanoma & 3,1 \\
\hline \multirow[t]{7}{*}{ Cocker } & 88 & 9,0 & $M: 61,36$ & Mastocitoma & 14,8 \\
\hline & & & $F: 38,64$ & Adenoma perianal & 10,2 \\
\hline & & & $\mathrm{NI}: 0$ & Tricoblastoma & 9,1 \\
\hline & & & & Melanoma maligno & 8,0 \\
\hline & & & & Lipoma & 8,0 \\
\hline & & & & $\begin{array}{c}\text { Histiocitoma cutâneo } \\
\text { canino }\end{array}$ & 6,8 \\
\hline & & & & Carcinoma perianal & 5,7 \\
\hline
\end{tabular}

$\bar{M}=$ macho; $\mathrm{F}=$ fêmea; $\mathrm{NI}$ = não informado; $\mathrm{CCE}$ = Carcinoma de células escamosas.

Os cães SRD foram os que mais apresentaram neoplasmas de pele $(n=272)$, seguidos pelos da raça Poodle $(n=97)$, Boxer ( $n=96)$, Cocker Spaniel $(n=88)$, Pastor Alemão $(n=57)$, Rottweiler $(n=50)$, Dachshund ( $n=29)$, Fila Brasileiro $(n=25)$, Labrador Retriever $(n=20)$, Yorkshire Terrier $(n=17)$, Pinscher $(n=17)$, Dogue Alemão $(n=16)$, Beagle $(n=14)$, Akita $(n=13)$, Lhasa Apso $(n=11)$, além de outros com $n<10$ (Fig.1). As raças Cocker Spaniel, Boxer e Poodle foram as mais afetadas por diversos tumores. O Quadro 3 demonstra a especificação dos tumores mais observados nessas raças.

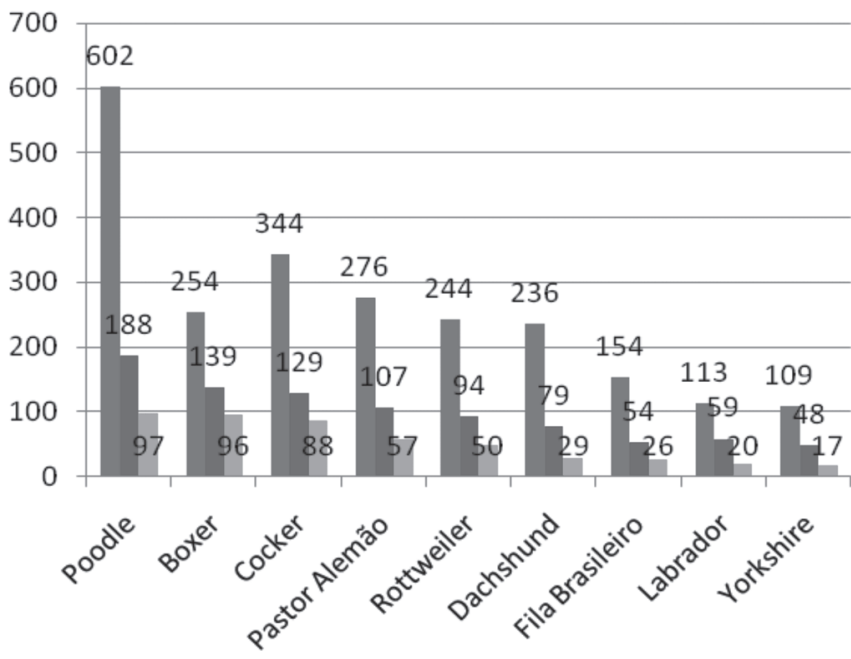

- Número total de amostras - Número de amostras de pele Número de neoplasias cutâneas

Fig.2. Número total de amostras, número de amostras de pele e número de neoplasmas cutâneos das principais raças no levantamento realizado no SPV da UFRGS, Porto Alegre, RS (2002-2007).

O número de amostras totais recebidas, amostras de pele e amostras com diagnóstico de neoplasma cutâneo em várias raças estão especificados na Figura 2.

\section{DISCUSSÃO}

Ao considerar-se o número total de amostras provenientes de cães que o Setor de Patologia Veterinária (SPV) recebeu durante o período deste levantamento, pode-se avaliar que a frequência de amostras com diagnóstico de neoplasma cutâneo foi de 20,3\% (1017/5.016). Dentre as amostras de pele de cães recebidas, 54,4\% (1.017/1.869) eram neoplasmas; esta frequência elevada para tumores cutâneos reforça a importância do estudo das neoplasias em dermatologia veterinária, visando, em primeira instância a diferenciação entre os vários tipos de neoplasmas e sua caracterização celular e em última análise, o aumento do bem-estar e qualidade de vida dos pacientes.

O número de amostras analisado foi importante, principalmente considerando que o período analisado foi relativamente curto. Informações mais específicas, tais como localização anatômica da neoplasia, não puderam ser incluídas no estudo devido à falta de dados no histórico encaminhado pelos veterinários requisitantes.

Observa-se marcada semelhança entre os resultados para neoplasmas cutâneos apresentados neste trabalho e aqueles obtidos por Souza et al. (2006), observando-se inclusive uma aproximação entre as frequências encontradas para os diferentes tipos de tumores junto à população canina. Foi observado um maior número de neoplasmas cutâneos malignos do que benignos, resultado também identificado por Kaldrymidou et al. (2002). Entretanto, difere de outros trabalhos de tumores cutâneos que apresentaram maior prevalência de benignos (Dobson et al. 2002, Bellei et al. 2006, Souza et al. 2006, Pakhrin et al. 2007). O neoplasma com maior frequência na população estuda- 
Quadro 4. Identificação dos cinco tumores cutâneos com maior prevalência encontradas neste levantamento e em estudos retrospectivos prévios

\begin{tabular}{|c|c|c|}
\hline Levantamento & Local & $\begin{array}{l}\text { Cinco tumores } \\
\text { mais frequentes }\end{array}$ \\
\hline Meirelles et al. 2009 & $\begin{array}{c}\text { Brasil } \\
\text { (Porto Alegre, RS) }\end{array}$ & $\begin{array}{c}\text { Mastocitoma } \\
\text { CCE } \\
\text { Lipoma } \\
\text { Adenoma perianal } \\
\text { Tricoblastoma }\end{array}$ \\
\hline Pakhrin et al. 2007 & Coreia & $\begin{array}{l}\text { Cistos epidermais } \\
\text { e foliculares } \\
\text { Lipoma } \\
\text { Mastocitoma } \\
\text { Histiocitoma } \\
\text { Tricoblastoma }\end{array}$ \\
\hline Souza et al. 2006 & $\begin{array}{c}\text { Brasil } \\
\text { (Santa Maria, RS) }\end{array}$ & $\begin{array}{c}\text { Mastocitoma } \\
\text { CCE } \\
\text { Adenoma perianal } \\
\text { Lipoma } \\
\text { Tricoblastoma }\end{array}$ \\
\hline Kaldrymidou et al. 2002 & Grécia & $\begin{array}{c}\text { Mastocitoma } \\
\text { Adenoma perianal } \\
\text { Lipoma } \\
\text { Histiocitoma } \\
-\end{array}$ \\
\hline Dobson et al. 2002 & Reino Unido & $\begin{array}{l}\text { Histiocitoma } \\
\text { Lipoma } \\
\text { Adenoma } \\
\text { Mastocitoma } \\
\text { Sarcoma }\end{array}$ \\
\hline Rothwell 1987 & Austrália & $\begin{array}{c}\text { Mastocitoma } \\
\text { Histiocitoma } \\
\text { Hemangiopericitoma } \\
\text { CCE } \\
\text { Fibrossarcoma }\end{array}$ \\
\hline Bostock 1986 & Reino Unido & $\begin{array}{c}\text { Mastocitoma } \\
\text { Adenoma perianal } \\
\text { Lipoma } \\
\text { Adenoma sebáceo } \\
\text { Fibrossarcoma }\end{array}$ \\
\hline
\end{tabular}

CCE $=$ Carcinoma de células escamosas .

da neste levantamento foi o mastocitoma. Este resultado está de acordo com outros estudos epidemiológicos realizados no Brasil (Bellei et al. 2006, Souza et al. 2006), na Grécia (Kaldrymidou et al. 2002), na Austrália (Rothwell 1987) e no Reino Unido (Bostock 1986) (Quadro 4).

A alta frequência de machos com neoplasma de glândula perianal pode ser explicada pelo fato das glândulas perianais serem andrógeno-dependentes. Pisani et al. (2006) comprovaram um maior número de receptores androgênicos em glândulas perianais hiperplásicas e encontraram os receptores em adenoma, epitelioma e carcinoma perianal. Esse achado reforça a importância da orquiectomia como parte do tratamento em machos.

O grupo de neoplasias que foi classificado como tumor mesenquimal maligno teve essa classificação pela observação das características mesenquimais das células. Porém, o grau de diferenciação e o padrão histológico analisado não permitiam dizer qual a classificação da neoplasia somente com a técnica de histologia. Em casos como es- tes, o indicado seria a realização de testes imuno-histoquímicos com anticorpos específicos para cada tipo celular. Muitas vezes, na prática, a observação da histologia, acrescida do comportamento maligno ou benigno do tumor é o suficiente para o clínico veterinário decidir sobre o tratamento que deve ser instituído para o animal e/ou optar por testes mais específicos. Todavia, por fatores econômicos e de disponibilização da técnica ou agilidade na entrega de resultados, estes não são rotineiramente requisitados.

A análise dos tumores apontando uma prevalência racial sugere a existência de predisposição genética de algumas raças para certos tumores cutâneos. Comparando-se as raças mais encontradas nos cinco principais tipos neoplásicos com dados da literatura revelam predisposições raciais em diferentes neoplasmas. Conhecida predisposição ocorre em cães da raça Boxer em apresentar mastocitoma (Gough \& Thomas 2004, Gross et al. 2005). Em nossa avaliação, o Boxer teve alta porcentagem de mastocitoma e foi a raça mais atingida por este tipo tumoral, confirmando esta observação. Raças predispostas a desenvolverem carcinoma de células escamosas são Keeshound, Schnauzer, Basset Hound, Collie e Boxer (Goldschmidt \& Hendrick 2002). Contudo, estas raças foram pouco prevalentes em nosso estudo. Em lipomas, as raças Labrador, Doberman, Schnauzer, Cocker Spaniel, Dachshund e Weimaraner são mencionadas como tendo maior predisposição (Gross et al. 2005). Entretanto, as raças citadas não foram encontradas ou apresentaram baixa ocorrência neste estudo. As duas principais raças com adenoma perianal foram a Poodle e o Cocker Spaniel. Conforme a literatura o Cocker Spaniel é citado como predisposto, assim como, o Husky Siberiano, Bulldog, Beagle, Samoieda (Goldschmidt \& Hendrick 2002, Gross et al. 2005). A raça Poodle e o Cocker Spaniel foram as mais atingidas por tricoblastoma, resultado também descrito na literatura (Goldschmidt \& Hendrick 2002, Gross et al. 2005). Considerando-se que na atualidade determinadas raças, especialmente as de companhia, de pequeno a médio porte, são preferenciais sobre outras e isto por si só seria suficiente para elevar o número de cães das raças apresentadas como mais prevalentes neste tipo de neoplasma.

A avaliação da frequência e compreensão do comportamento biológico dos neoplasmas mais comuns em cães são fundamentais para direcionar a pesquisa por novos tratamentos, que em auxiliando os veterinários que atuam na clínica médica e cirúrgica, melhorarão indiscutivelmente a saúde de seus pacientes. Considerando-se a diversidade de características relacionadas a cada tipo de neoplasma, releva-se a importância na sua caracterização. Adicionalmente, a expansão de técnicas diagnósticas que possam contribuir de modo efetivo para a detecção e controle precoce de neoplasias cutâneas nos cães constituise num aspecto decisivo e facilitador no tratamento destas doenças.

Agradecimentos.- Aos colegas, técnicos e amigos do Setor de Patologia Veterinária da UFRGS pelo auxílio técnico. Ao Conselho Nacio- 
nal de Desenvolvimento Científico e Tecnológico (CNPq) pelo auxílio financeiro durante execução deste trabalho.

\section{REFERÊNCIAS}

Bellei M.H.M., Neves D.S., Gava A., Liz P.P. \& Pilati C. 2006. Prevalência de neoplasias cutâneas diagnosticadas em caninos no estado de Santa Catarina, Brasil, no período entre 1998 e 2002. Revta Ciênc. Agrovet. 5:73-79.

Bostock D.E. 1986. Neoplasms of the skin and subcutaneous tissues in dogs and cats. Brit. Vet. J. 142:1-19.

Dobson J.M., Samuel S., Milstein H., Rogers K. \& Wood J.L.N. 2002. Canine neoplasia in the UK: Estimates of incidence rates from a population of insured dogs. J. Small Anim. Pract. 43:240-246.

Goldschmidt M.H. \& Hendrick M.J. 2002. Tumors of the skin and soft tissues, p.45-117. In: Meuten D.J. (Ed.), Tumors in Domestic Animals. $4^{\text {th }}$ ed. Blackwell, lowa.

Goldschmidt M.H. Dunstan R.W., Stannard A.A., von Tscharner C., Walder E.J. \& Yager J.A. 1998. Histological Classification of Epithelial and Melanocytic Tumors of the Skin of Domestic Animals. $2^{\text {nd }}$ ed. Armed Forces Institute of Pathology, Washington. 105p.

Gough A. \& Thomas A. 2004. Breed Predispositions to Diseases in Dogs and Cats. Blackwell, Oxford, p.33-35.

Gross T.L., Ihrke P.J., Walder E.J. \& Affolter V.K. 2005. Skin Diseases of the Dog and Cat: Clinical and histopathologic diagnosis. $2^{\text {nd }}$ ed. Blackwell, Oxford, p. 561-865.

Kaldrymidou H., Leontides L., Koutinas A.F., Saridomichelakis M.N. \& Karayannopoulou M. 2002. Prevalence, distribution and factors associated with the presence and the potential for malignancy of cutaneous neoplasms in 174 dogs admitted to a clinic in Northern Greece. J. Vet. Med. A. 49:87-91.

Medleau L. \& Hnilica K.A. 2003. Dermatologia de Pequenos Animais: Atlas colorido e guia terapêutico. $2^{\text {nd }}$ ed. Roca, São Paulo, p.395-398.

Murphy S. 2006a. Skin neoplasia in small animals. 1. Principles of diagnosis and management. In Pract. 28:266-27.

Murphy S. 2006b. Skin neoplasia in small animals. 3. Common canine tumours. In Pract. 28:398-402.

Pakhrin B., Kang M., Bae I., Park M., Jee H., You M., Kim J., Yoon B., Choi Y. \& Kim D. 2007. Retrospective study of canine cutaneous tumors in Korea. J. Vet. Sci. 8:229-236.

Patnaik A.K., Ehler W.J. \& MacEwen E.G. 1984. Canine cutaneous mast cell tumor: morphologic grading and survival time in 83 dogs. Vet. Pathol. 21:469-474.

Pisani G., Millanta F., Lorenzi D., Vannozzi I. \& Poli A. 2006. Androgen receptor expression in normal, hyperplastic and neoplastic hepatoid glands in the dog. Res. Vet. Sci. 81:231-236.

Rothwell T.L.W. 1987. Skin neoplasms of dogs in Sydney. Aust. Vet. J. 64:161-164.

Souza T.M., Fighera F.A., Irigoyen L.F. \& Barros C.S.L. 2006. Estudo retrospectivo de 761 tumores cutâneos em cães. Ciência Rural 36:555-560.

Stannard A.A. \& Pulley L.T. 1978. Tumors of the skin and soft tissues, p.16-71. In: Moulton J.E. (Ed.), Tumors of Domestic Animals. $2^{\text {nd }}$ ed. University of California Press, London.

Yager J.A. \& Scott D.W. 1992. The skin and appendages, p.531-738. In: Jubb K.U.F., Kennedy P.C. \& Palmer N. (Eds), Pathology of Domestic Animals. Vol.1. $4^{\text {th }}$ ed. Academic Press, London. 